\title{
Gamma-ray emission from supermassive black hole binary OJ 287
}

\author{
Vera Yu. Sinitsyna ${ }^{1, *}$ and Vera G. Sinitsyna ${ }^{1, * *}$ \\ ${ }^{1}$ P.N. Lebedev Physical Institute, Moscow, Russia
}

\begin{abstract}
OJ 287 is one of the most studied BL Lacs with very long optical measurements which spectrum has been well measured through radio to X-rays. OJ 287 is supposed to be a binary black hole system. Its secondary black hole passes the accretion disk of the primary black hole and produces two impact flashes per 12-year period. Observations of OJ 287 in the $\mathrm{GeV}-\mathrm{TeV}$ energy range reveal the variable $\gamma$-ray flux connected with the flare activity of this object. The spectral energy distributions of BL Lac objects consist of two broad peaks. The Inverse Compton emission of the relativistic electrons in the jet or combined with an external Compton mechanism are considered in the leptonic scenario of second, higher frequency spectrum part generation. The last one supposes the existence of the external to jet photon cloud. Also, the second spectrum part is supposed to be generated due to the acceleration of the cosmic ray hadrons in expanding shock produced by outflow, which then collides with the wind of the primary black hole. The detection of $\mathrm{GeV}-\mathrm{TeV}$ energy fluxes can help find the configuration parameters of the two-black hole system.
\end{abstract}

\section{Introduction}

BL Lacs are a high and rapidly variable subclass of radio-loud, active galactic nuclei (AGN) characterized by non-thermal spectra extending from radio to high and very high energy $\gamma$ rays. The short time variability of a day or even minute timescale and the detection at $\mathrm{TeV}$ energies points to the origin of emission from the relativistic jets directed to the observer sightline [1]. It suggests that the emission region is located at the parsec scales from the central powering engine. The spectral energy distribution of the BL Lacs is of a two-hump shape with a first peaking at a low-energy in infrared to X-ray range and a second at high energy $\gamma$-rays. The production of these emission components and their connection is widely studied. The origin of the high energy emission is a matter of great interest and involves both leptonic and hadronic models. So, the observations of powerful BL Lac-type active galactic nuclei can provide information for the models of described the jet dynamic in AGNi and their emission in the wide range of the electromagnetic spectrum.

\section{OJ 287}

OJ 287 is one of the most studied blazars of low-frequency peaked BL Lac type which spectrum has been well measured through radio [2], [3] to X-ray [4], [5] and optical bands. The most outstanding characteristic of OJ 287 is its 12 year period, which is discovered throughout the periodic outburst in the optical range [4] and has also been confirmed in the X-ray band. This periodic behavior is supposed to be due to the possible presence of a binary supermassive black hole in which a secondary black hole passes the accretion disk of the primary

\footnotetext{
*e-mail: sinicinavy@lebedev.ru

**e-mail: sinits@sci.lebedev.ru
} 

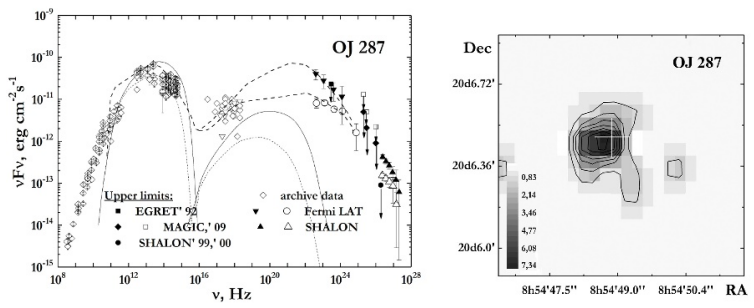

Figure 1. Spectral energy distribution of OJ 287.

$\triangle$ show the average spectrum;

$\Delta$ show the flaring spectra from the

SHALON data. Emission map of

OJ 287 by SHALON (grey scale).

black hole and produces two impact flashes per period. At high energies, OJ 287 has been detected with $\mathrm{GeV}$ emissions by EGRET [10], Fermi-LAT[11], and proposed to be a TeV source.

As mentioned above, the spectral energy distributions of blazars consist of two broad peaks. The first, lower frequency peak is due to the synchrotron emissions of relativistic electrons in the jet. Then in the leptonic scenarios, it is supposed that the second, higher frequency peak is to be due to the Inverse Compton emissions of the same electrons on the surrounding photon fields [6].The inverse Compton scattering of soft photons external to the jet is also considered [7]. The hadronic scenarios invoke proton-synchrotron and proton-photon cascade processes (e.g. [8]), and also cosmic ray accelerated in the shock generated by outflows in the interactions of the parts of the binary black hole system [9].

Under the SHALON program of long-term studies of active galactic nuclei at TeV energies, observations of OJ 287 are being carried out with our experiment. OJ 287 was observed by SHALON in 1999, 2000, 2008 - 2010. The observations of 1999 and 2000 years don't reveal a $\gamma$-ray flux from the position of OJ 287, but only an upper limit of $<1.1 \times 10^{-13} \mathrm{ph} \mathrm{cm}^{-2} \mathrm{~s}^{-1}$. In observations of 2008, 2009 and 2010 the weak $\gamma$-ray flux was detected [12]. An excess corresponding to a $6.9 \sigma$ [13] was determined. The observed integral flux at $>800 \mathrm{GeV}$ is $(0.26 \pm 0.07) \times 10^{-12} \mathrm{ph} \mathrm{cm}^{-2} \mathrm{~s}^{-1}$. The measured overall energy spectrum of OJ 287 can be fitted by a power-law with an exponential cutoff described by $d N / d E=(1.95 \pm 0.16) \times(E / 1 \mathrm{TeV})^{-2.37 \pm 0.20} \times \exp (-E /(7.8 \pm 1.4)) \times 10^{-13} \mathrm{TeV}^{-1} \mathrm{~cm}^{-2} \mathrm{~s}^{-1}$.

Figure 1 presents the spectral energy distribution of the $\gamma$-ray emission from OJ 287 by SHALON ( $\triangle$ and $\Delta$ ) compared to other experimental data [14], [10], and theoretical predictions from [7], [11] and also [14]. The open triangles at TeV energies on Fig. 1 are the average spectrum of OJ 287 from SHALON experiment; an upper limit at $>0.8 \mathrm{TeV}$ corresponds to SHALON observations in 1999, 2000. The black triangles present the $\gamma$-ray spectrum at the increased flux period of 2010. The flux increase over the detected average flux was found on November 14,15, and December 4, 5 of 2010. The average value of increase is $(0.63 \pm 0.15) \times 10^{-12} \mathrm{ph} \mathrm{cm}^{-2} \mathrm{~s}^{-1}$ (at $6.2 \sigma$ [13] level), with the softer energy spectrum with a power law with an index of $-2.95 \pm 0.21$. Also, the high flux was detected on November 16, 2009. The duration of the flux increase in December 2010 was 2 days with a peak intensity of $(1.16 \pm 0.51) \times 10^{-12} \mathrm{ph} \mathrm{cm}^{-2} \mathrm{~s}^{-1}$. No duration of intervals of flux increase was found in 2009 because the observations were interrupted due to weather conditions. To find correlations of the emissions in various energy ranges, we compared the $\mathrm{TeV} \gamma$-ray fluxes from OJ287 by SHALON with those at MeV-GeV range by the Fermi-LAT experiment (see Fig. 2) and data in R-band (Suhora Astronomical Observatory) [15].

\section{Discussion}

It was investigated in [9] a hadronic origin for the X-ray and $\gamma$-ray counterparts of one of the major optical flares of OJ 287 occurring after the second lighter black hole passing through the accretion disc of the heavier one. The cosmic ray proton acceleration occurs in the shock generated by outflows interactions with the active galactic nucleus wind of the heavier black hole. A hadronic emission component is compatible with the $\gamma$-ray data, but to reproduce the $\mathrm{TeV}$ energy spectrum, optical depth for $\mathrm{p}-\mathrm{p}$ annihilation should be lower. 


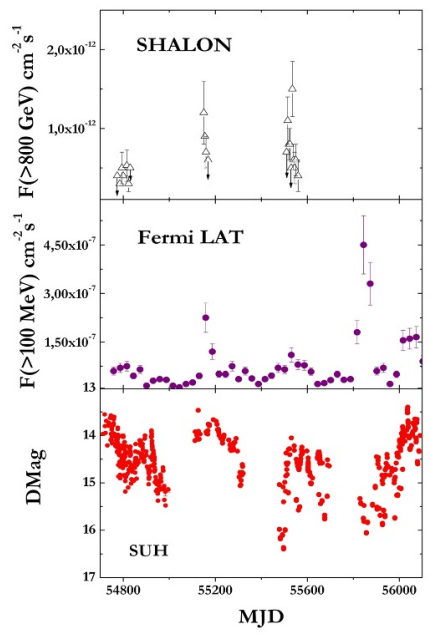

In [7], the model used the combination of self-Compton and external Compton mechanisms was applied to describe the SED of OJ 287 during a major $\gamma$-ray flare of the 2009 year (Fig. 1, dashed lines) also detected at TeV energies. The size of the emission region found to reproduce the observed spectral energy distribution is about $9 \mathrm{pc}$, consistent with one revealed [17] from the relation of flares in the millimeter, optical, and $\mathrm{GeV}$ energy range.

It was found in [17] that the two $0.1-200 \mathrm{GeV}$ flares in OJ 287, as well as two optical flares are related to the two millimeter-wave flares that originated in the second of two $>14$ pc separated features in the jet. Such multiwavelength behavior can be explained if the $\gamma$ rays are produced due to the synchrotron self-Compton scattering of optical photons from the flares. It is proposed that the flare is launched by the interaction of moving plasma blobs with a standing shock. The flare correlations can indicate that the emission observed by SHALON is a $\mathrm{TeV}$ particle population also formed the spectrum viewed by Fermi-LAT, which produced by the synchrotron self-Compton process occurs in the jet away from the central core.

\section{Conclusions}

Experimental data on $\mathrm{TeV} \gamma$-ray emission from OJ 287 of the different stages, including the flaring activity period of 2008 - 2010 yy are obtained in the SHALON long-term observations and compared with ones at the GeV range by Fermi-LAT and optical data from Suhora Astronomical Observatory. The correlation of the flares in optical, $\mathrm{GeV}, \mathrm{TeV}$ energies may indicate that the observed spectral energy distribution in $\mathrm{TeV}$ energies can be described in terms of the combination of self-Compton and external Compton mechanisms and connected with the interaction of moving plasma blobs launched in the jets with a standing shock.

\section{References}

[1] L. Dondi, G. Ghisellini, Mon. Not. R. Astron. Soc. 273, 583 (1995)

[2] E. Idesawa et al., Publ. Astron. Soc. Jpn. 49, 631 (1997)

[3] H. Seta et al., Publ. Astron. Soc. Jpn. 61, 1011 (2009)

[4] E. Valtaoja et al., Astrophys. J. 531, 744 (2000)

[5] C.E. Tateyama, K.A. Kingham, Astrophys. J. 608, 149 (2004)

[6] G. Ghisellin et al., Mon. Not. R. Astron. Soc. 301, 451 (1998)

[7] P. Kushwaha, S. Sahayanathan, K.P. Singh, Mon. Not. R. Astron. Soc. 433, 2380 (2013)

[8] A. Mücke, R.J. Protheroe, R. Engel et al., Astropart. Phys. 18, 593 (2003)

[9] J.C. Rodríguez-Ramírez et al., Mon. Not. R. Astron. Soc. 498, 5424 (2020)

[10] R.C. Hartman et al., ApJS 123, 79 (1999)

[11] A.A. Abdo et al., Astrophys. J. 700, 597 (2009); ibid. 715, 429, 132 (2010) 
[12] V.G. Sinitsyna et al., Proc. of 32nd ICRC, Beijing, China, 2011; 8/11, 26 (2011)

[13] T.-P. Li \& Y.-Q. Ma, Astrophys. J. 272, 317 (1983)

[14] M. Hayashida et al., Proc. of 31st ICRC, Lodz, Poland, 2009;

[15] P. Pihajoki, M. Valtonen et al., Astrophys. J. 764, 5 (2013)

[16] M. Ackermann et al., Astrophys. J. 810, 14 (2015)

[17] I. Agudo, S.G. Jorstad, A.P. Marscher et al., Astrophys. J. Lett. 726, L13 (2011) 